\title{
Structural and Optical Investigation of Novel Europium Hybrid Organic Complex for OLEDs and Solid State Lighting
}

\author{
Dipti Chitnis ${ }^{1}$, N. Thejo Kalyani ${ }^{2, *}$, S.J. Dhoble ${ }^{1}$ \\ ${ }^{1}$ Department of Physics, RTM Nagpur University, Nagpur-440033, India \\ ${ }^{2}$ Department of Applied Physics, Laxminarayan Institute of Technology, Nagpur-440033, India \\ *Corresponding author: thejokalyani@rediffmail.com
}

\begin{abstract}
We report the design and synthesis of volatile europium $\beta$-diketonate Eu(dmh) $)_{3}$ phen organic hybrid complex by precipitation technique. A series of complexes were synthesized for different $\mathrm{pH}$ values, ranging between 8.0 and 6.0 maintaining stoichiometric ratio. Among all the synthesized complexes, $\mathrm{Eu}(\mathrm{dmh})_{3} \mathrm{phen}$ with $\mathrm{pH}$ 7 exhibits red intense emission at $613 \mathrm{~nm}$ with a sharp spectral bandwidth of $5 \mathrm{~nm}$ when excited at a wavelength of $372 \mathrm{~nm}$,infering that the synthesized complex is $\mathrm{pH}$ sensitive. This complex is further assessed for structural, thermal and luminescence behavior in various solvents. X-ray diffractogram reveals its crystalline nature and FTIR confirms the complete formation of the complex revealing the presence of TTA and bipy structure in the metal complex. TGA/DTA exhibits good thermal stability, which is highly essential for fabricating organic light emitting diodes (OLEDs) for solid state lighting. Hypsochromic shift was observed in the optical absorption spectra of $\mathrm{Eu}(\mathrm{dmh})_{3}$ phen, when the solvent is changed from basic to acidic media.
\end{abstract}

Keywords: biodegradable, europium, solid state lighting, OLEDs

Cite This Article: Dipti Chitnis, N. Thejo Kalyani, and S.J. Dhoble, "Structural and Optical Investigation of Novel Europium Hybrid Organic Complex for OLEDs and Solid State Lighting.” Journal of Materials Physics and Chemistry, vol. 5, no. 1 (2017): 1-10. doi: 10.12691/jmpc-5-1-1.

\section{Introduction}

Since time immemorial, there is an incredible change in lighting technology, as it plays a significant factor contributing to the quality and productivity of human life. Though fire was used by our primate ancestors 2-6 million years ago, it is still thought of as an exemplary human invention [1]. But till date the so developed technologies face many challenges in order to offer ecofriendly climate and energy efficient solid state lighting (SSL). Billions of people around the world, including the poorest and most vulnerable small holders still depend on fuel based lighting, which is climate hazardous. Hence, there is growing focus on adoption of technology for improvising lighting techniques, with an agenda of green lighting to ensure light for all. For SSL, highly intense and narrow peak emission of the primary colours (RGB) is essential. However, red light emission is considered as the weakest one because the transition corresponding to red light occurs at a comparatively small energy band gap, which is hard to match the energy level of carrier transmission layer [2]. This drawback can be overcome to certain extent by employing europium complexes in view of their high photo luminescent (PL) efficiency and the red-emission ability that are widely exploited in full-color displays. At present, research activity involving the design and construction of novel
$\mathrm{Eu}^{3+}$ complexes for the light-emitting device is prevalent because of their sharp emission peak at $612 \mathrm{~nm}[3,4]$. $\beta$-diketones (1,3-diketones) with rare-earth ions are the most popular and the most intensively investigated rare earth coordination compounds [5]. This popularity is partially due to the fact that many different $\beta$-diketones are commercially available and the fact that the synthesis of the corresponding rare-earth complexes is relatively easy [6]. However, the main drive for the intense research activity on the rare-earth $\beta$-diketonates was and is still continued due to their potential of being used in several applications [7,8]. Hence, it proposed to design, synthesize and characterize novel europium complex with $\mathrm{dmh}$ and phen as ligands by precipitation technique.

\section{Experimental}

Chemicals of analytical reagents (AR) grade were purchased from Sigma Aldrich and Across organics. All reactions were carried out with dry, freshly distilled solvents under anhydrous conditions or in an inert atmosphere.

\subsection{Reagents and Solvents}

Starting materials used for the synthesis of $\mathrm{Eu}(\mathrm{dmh})_{3}$ phen complex are europium chloride hexahydrate $\left(\mathrm{EuCl}_{3} \cdot 6 \mathrm{H}_{2} \mathrm{O}\right)$, [Across chemicals] Purity - 99.99\%, M.Wt = $366.40 \mathrm{~g} / \mathrm{mol}$. 
2,6-Dimethyl-3,5-heptanedione, [pale yellow liquid] $\left(\mathrm{C}_{9} \mathrm{H}_{16} \mathrm{O}_{2}\right)$ [Across chemicals] Purity - 99\%; M.Wt $=127.13 \mathrm{~g} / \mathrm{mol}$. Phen- 1,10 Phenanthroline, $\left(\mathrm{C}_{12} \mathrm{H}_{8} \mathrm{~N}_{2} \cdot \mathrm{H}_{2} \mathrm{O}\right)$ [Fisher scientific] purity $-99.9 \%$, M.Wt $=198.23 \mathrm{~g} / \mathrm{mol}$ Ethanol absolute $\left(\mathrm{C}_{2} \mathrm{H}_{5} \mathrm{OH}\right)$, purity $-99.98 \%$, M.Wt $=46.07 \mathrm{~g} / \mathrm{mol}$, chloroform $\left(\mathrm{CHCl}_{3}\right)$ [Qualigens company] purity - $99.7 \%$, M.wt.= 119.38, Toulene $\left(\mathrm{C}_{6} \mathrm{H}_{5} \mathrm{CH}_{3}\right)$, M.wt $=92.14$ gm purity $99.9 \%$, refractive index is $1.493-1.497$, b.p $(92 \%)=110-$ $111^{\circ} \mathrm{C}$; Tetra hydro Furan(THF) $\left(\mathrm{C}_{4} \mathrm{H}_{8} \mathrm{O}\right)$, M.wt $=72.11$ gm purity $-99.9 \%$, refractive index at $20^{\circ} \mathrm{C}$ is $1.407-1.409$, boiling range $65-67^{\circ} \mathrm{C}$, Acetic acid $\left(\mathrm{CH}_{3} \mathrm{COOH}\right)$ [sd fine chemicals] purity - 99.5\%, M.wt. $=60.05$, Potassium hydroxide $(\mathrm{KOH})$, purity - 99.9\% and double distilled water.

\subsection{Structure and Synthesis of Organic Complexes}

The chemical structure of dmh, phen, Eu(dmh) $)_{3}$ phen are shown in Figure 1(a) (b) and (c), respectively. Synthesis scheme of Eu $(\mathrm{dmh})_{3}$ phen complex is shown in Figure 2 It can be clearly stated that $\mathrm{Eu}^{3+}$ is associated with three molecules of dmh and one molecule of phen. $\mathrm{Eu}^{3+}$ has 8 co-ordinates (6 with $\mathrm{dmh}$, which are shown to the left of $\mathrm{Eu}^{3+}$ while the other two with phen on the right side). For the formation of $\mathrm{Eu}(\mathrm{dmh})_{3}$ phen complex, the stichiometry of chemical compounds are taken in 1:3:1 ratio.

\subsection{Synthesis Procedure}

Step 1: $\operatorname{dmh}(6.63 \mathrm{mmol}=0.842 \mathrm{gm})$ and phen $(2.21$ $\mathrm{mmol}=0.438 \mathrm{gm}$ ) were dissolved in $20 \mathrm{ml}$ ethanol (Sol I).

Step 2: Later, $\mathrm{EuCl}_{3} \cdot 6 \mathrm{H}_{2} \mathrm{O}$ (2.21 mmol = $\left.0.809 \mathrm{gm}\right)$ was separately dissolved in $10 \mathrm{ml}$ double distilled water in another beaker (Sol II).
Step 3: Both the solutions were mixed and the $\mathrm{pH}$ of the resultant solution was found to be in between 0.25 0.35 . In order to obtain a neutral solution with $\mathrm{pH}=7$, $\mathrm{KOH}$ solution is added drop by drop into the resultant solution.

Step 4: The solution thus obtained was heated at $60^{\circ} \mathrm{C}$ on a hot plate with continuous stirring with a magnetic stirrer for 1 hour.

Step 5: The precipitate so obtained was filtered and washed with double distilled water twice or thrice.

Step 6: The complex was dried at room temperature and then eventually in the hot air oven at $80^{\circ} \mathrm{C}$ for two hours [9].

Similarly, series of complexes were prepared by increasing $\mathrm{pH}$ to 8.0 from 7.0 and also by decreasing the $\mathrm{pH}$ to 6.0 from 7.0 at an interval of $0.5 \mathrm{pH}$. Pictorial demonstration of synthesis procedure of $\mathrm{Eu}(\mathrm{dmh})_{3}$ phen complex is shown in Figure 3.

\section{Result and Discussion}

Structural properties of Eu(dmh) $)_{3}$ phen are evaluated by wide angle X-ray diffraction on Philips Analytical X' Pert Pro Powder Diffractometer, while Fourier Transform Infrared spectra and thermal properties of the synthesized complexes are explored on Perkin Elmer-Spectrum one and Perkin Elmer Diamond TGA/DTA analyzer, respectively. Optical absorption and emission spectra were carried out on HR 4C $4568 \mathrm{UV}$-Vis spectro photophotometer and Humamatsu F-4500 spectroflurometer, respectively. Among all the synthesized complexes, maximum photoluminescence intensity was obtained from $\mathrm{Eu}(\mathrm{dmh})_{3}$ phen with $\mathrm{pH}=7.0$. Hence this complex is considered for further characterizations such as XRD, TGA/DTA, FTIR, optical absorption spectra in acidic and basic media.

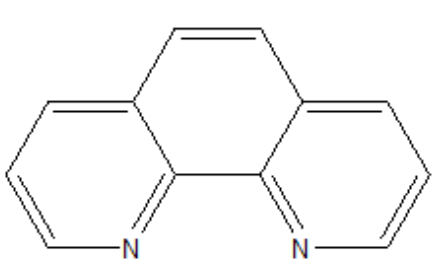

(a)

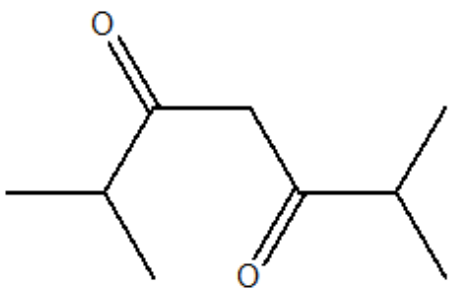

(b)

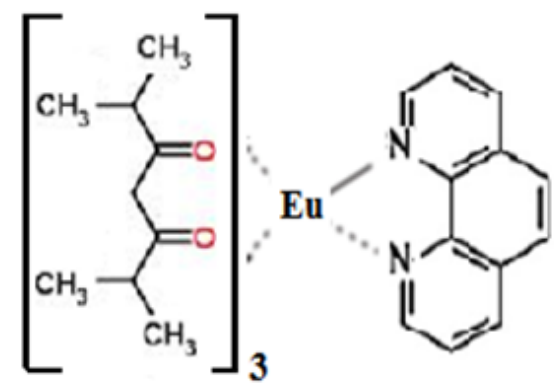

(c)

Figure 1. The chemical structure of (a) 1,10 Phenanthroline (b) 2,6-Dimethyl-3,5-heptanedione (c) Eu(dmh) 3 phen<smiles>Clc1cc(Cl)cc(Cl)c1</smiles><smiles>CCCCCCCCCCCC(C)C</smiles>

\section{$\mathrm{CH}_{3} \mathrm{CH}_{2} \mathrm{OH}$; $\mathrm{KOH}$}

distilled water at $60^{\circ} \mathrm{C}$ for $1 \mathrm{Hr}$

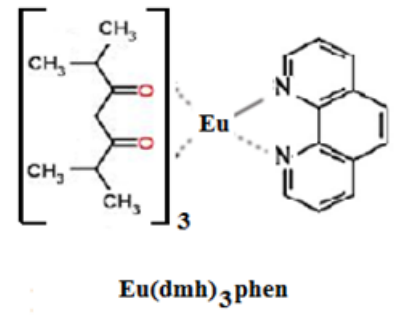

Figure 2. Synthesis route of $\mathrm{Eu}(\mathrm{dmh})_{3}$ phen complex by solution technique 


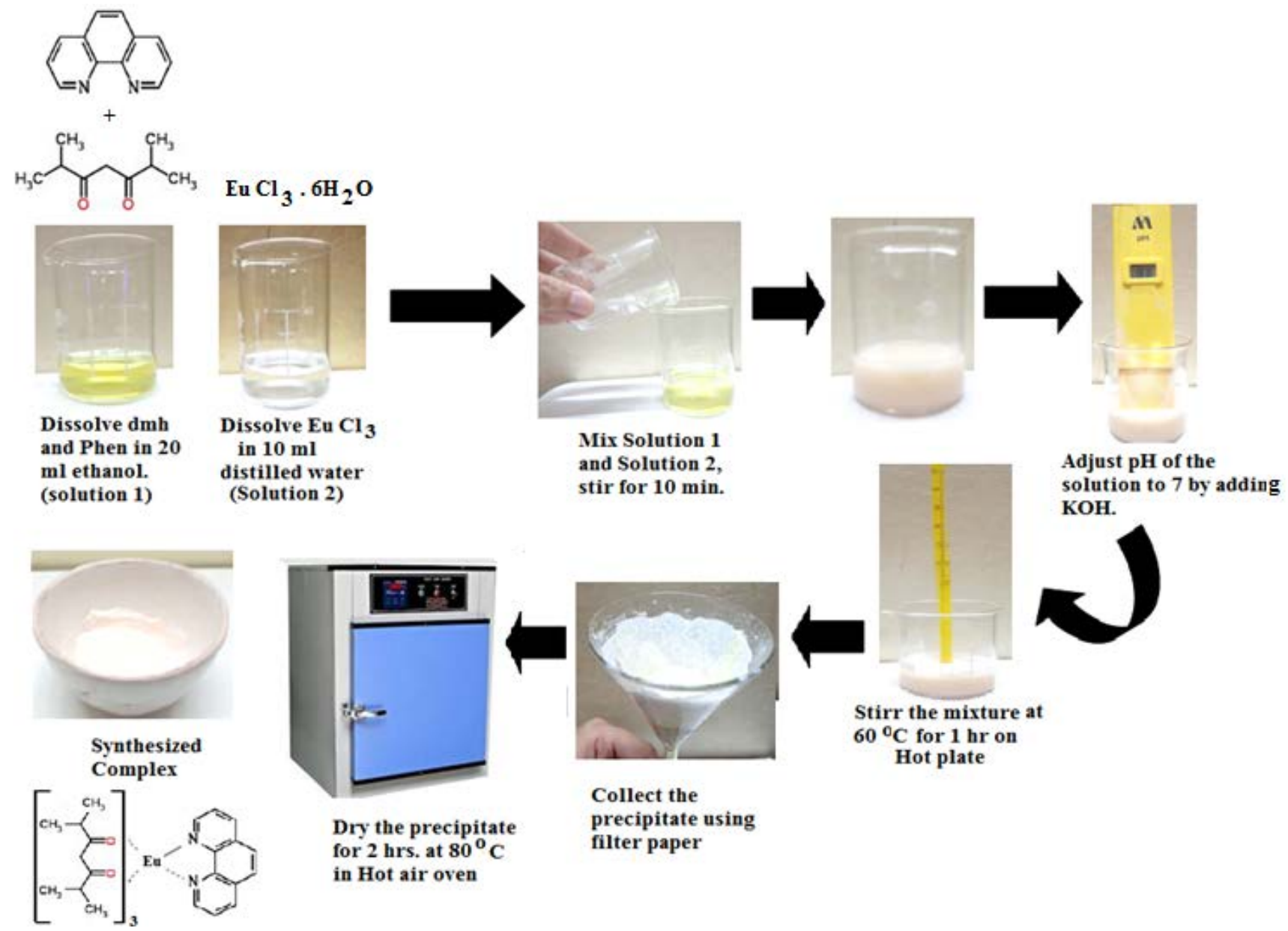

Figure 3. Pictorial demonstration of synthesis procedure of $\mathrm{Eu}(\mathrm{dmh})_{3}$ phen complex

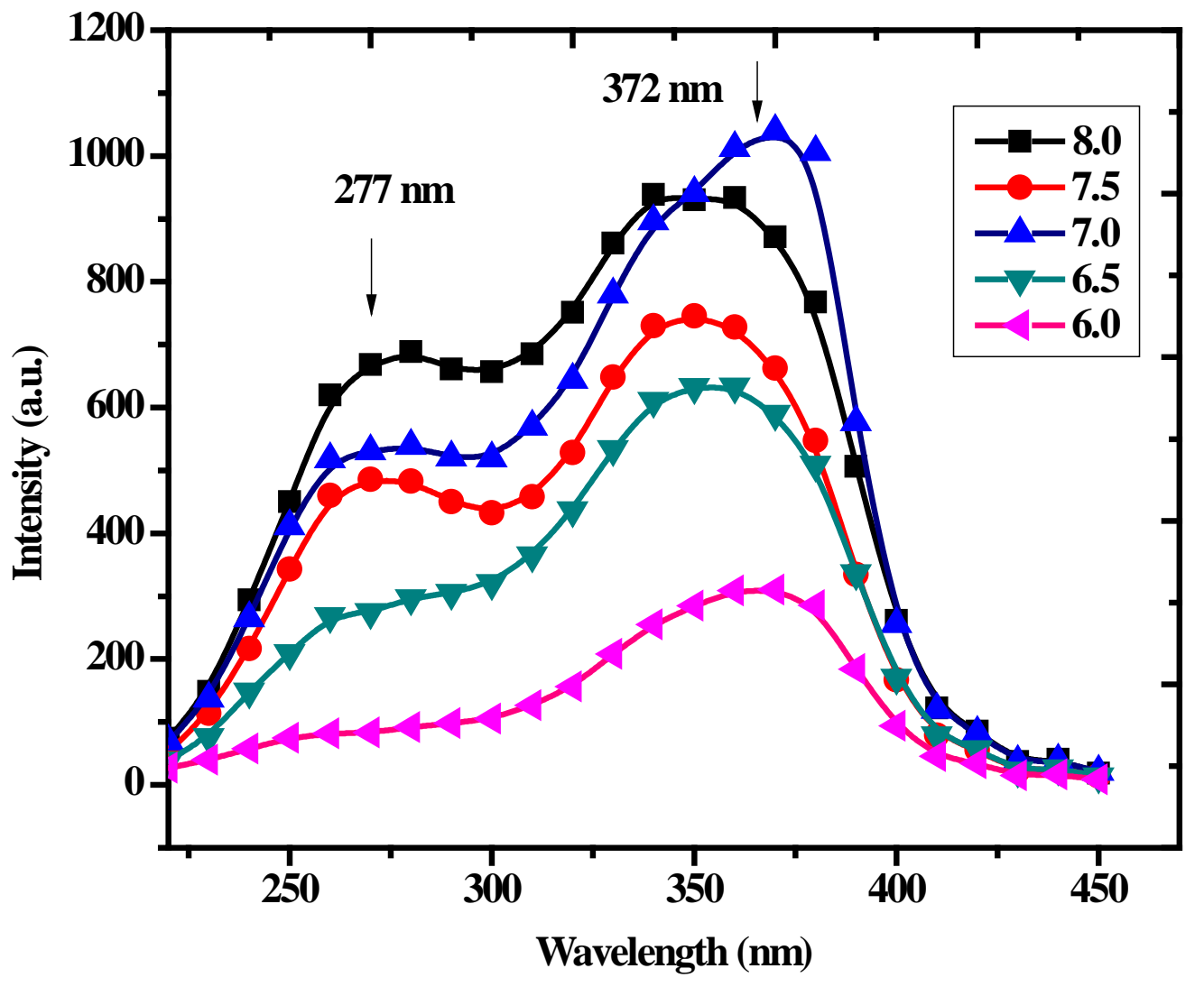

Figure 4. Excitation spectra of $\mathrm{Eu}(\mathrm{dmh})_{3} \mathrm{phen}$ complex for different values of $\mathrm{pH}$ 


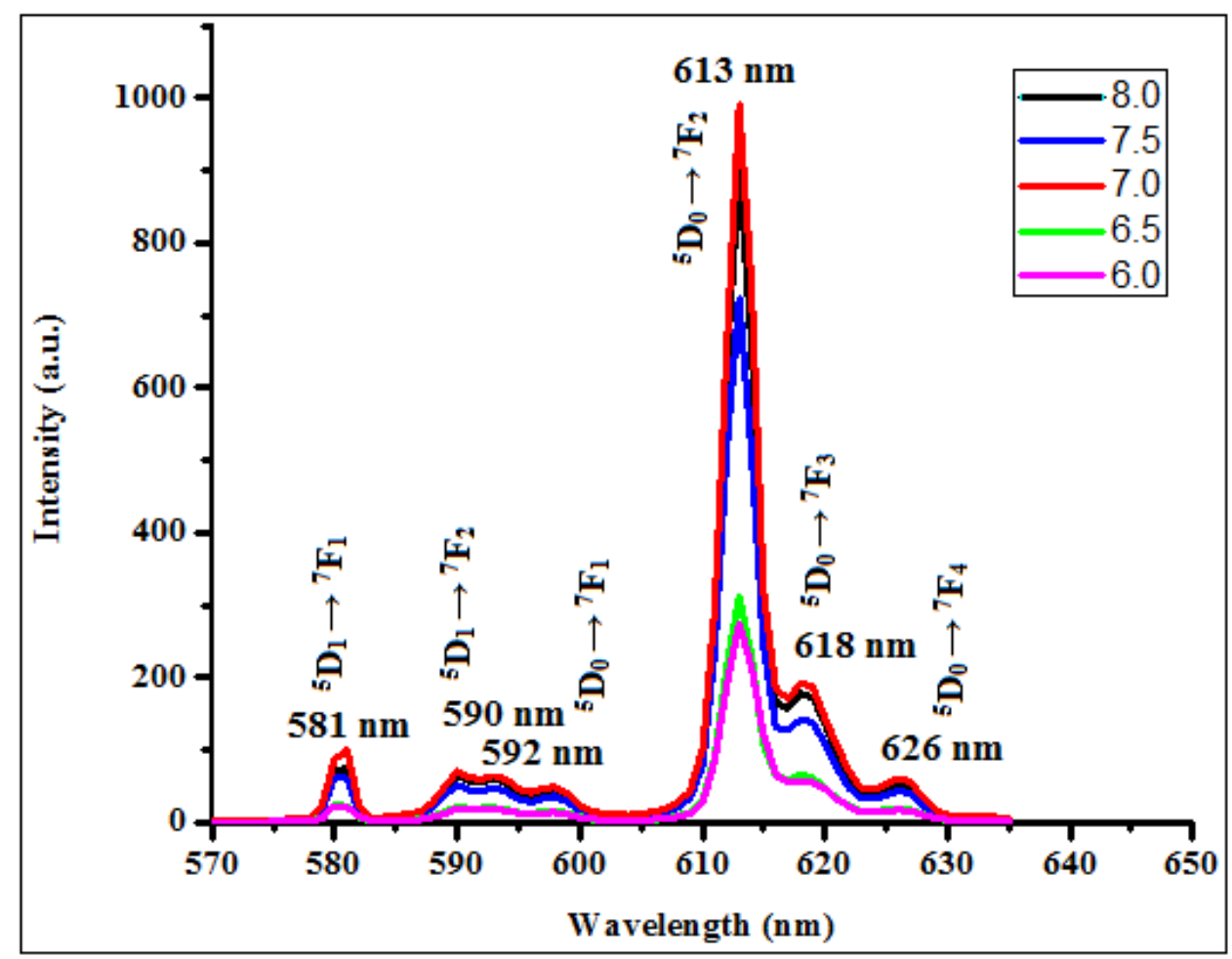

Figure 5. Emission spectra of $\mathrm{Eu}(\mathrm{dmh})_{3}$ phen complex for different values of $\mathrm{pH}$

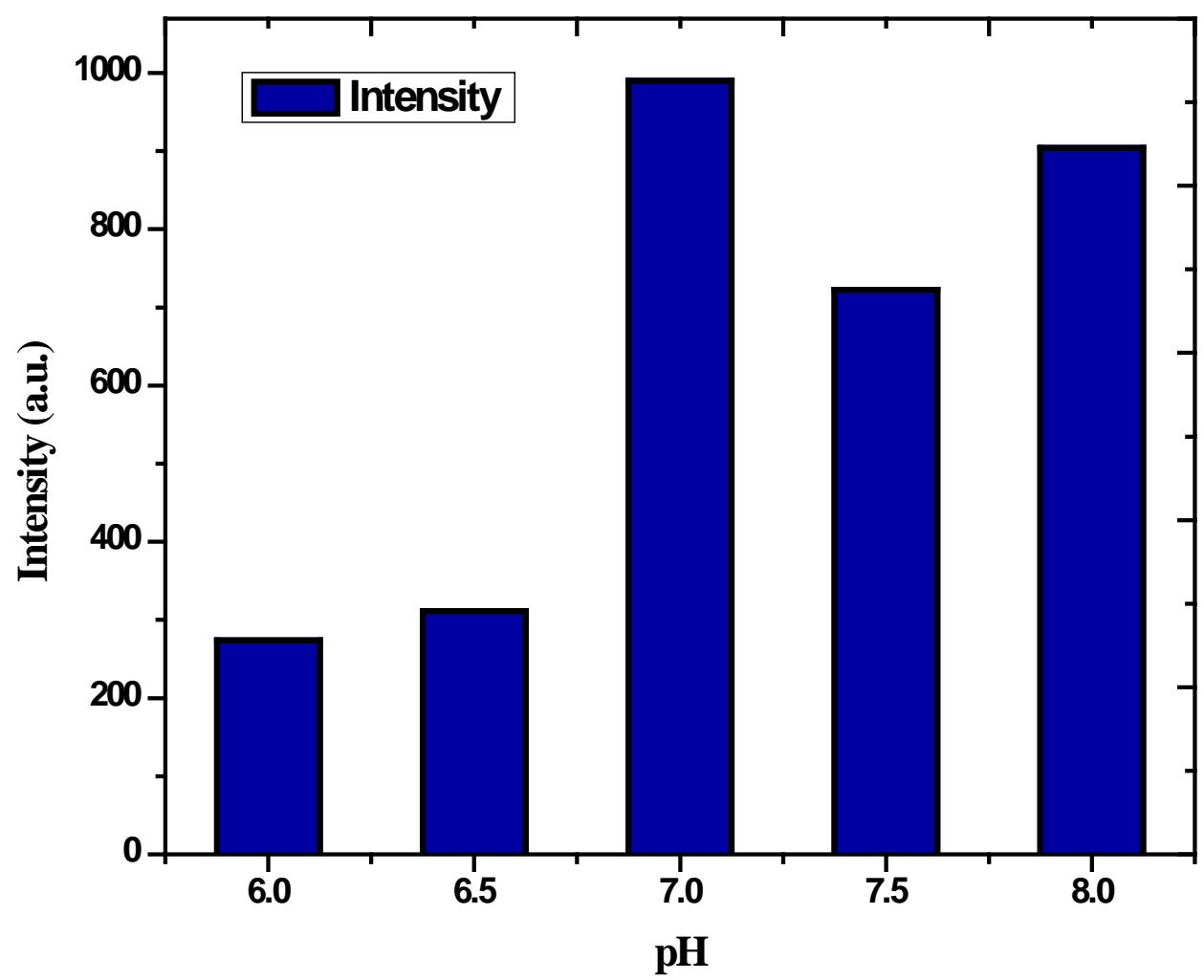

Figure 6. The variation of emission intensity of complex with $\mathrm{pH}$

\subsection{Photoluminescence Spectra}

The excitation and emission spectrum of Eu(dmh) $)_{3}$ phen complexes at $\mathrm{pH}$ ranging from 8.0 to 6.0 is shown in Figure 4 and Figure 5, respectively. The complexes were excited in the wavelength range of $250 \mathrm{~nm}$ to $500 \mathrm{~nm}$ monitoring emission at $613 \mathrm{~nm}$. Variation in intensity of red light emission was observed with change in $\mathrm{pH}$.
Emission spectra revealed that $\mathrm{Eu}(\mathrm{dmh})_{3}$ phen exhibits a sharp spectral emission centered at $613 \mathrm{~nm}$ when excited at $372 \mathrm{~nm}$ wavelength. PL emission spectrum also displays weak emission peaks at 581nm, $590 \mathrm{~nm}$ and 592 $\mathrm{nm} 618 \mathrm{~nm}$ and $626 \mathrm{~nm}$.

The emission spectra of the Eu(dmh) $)_{3}$ phen complex showed the highest intensity at $613 \mathrm{~nm}$, which corresponds to the ${ }^{5} \mathrm{D}_{0} \rightarrow{ }^{7} \mathrm{~F}_{2}$ electric dipole transition. The 
intensity of the electric dipole transition ${ }^{5} \mathrm{D}_{0} \rightarrow{ }^{7} \mathrm{~F}_{2}$ transition is much stronger than the magnetic dipole allowed ${ }^{5} \mathrm{D}_{0} \rightarrow{ }^{7} \mathrm{~F}_{1}$ transition, indicating that $\mathrm{Eu}^{3+}$ ions in these systems occupy very low symmetric sites [10]. This hyper sensitive ${ }^{5} \mathrm{D}_{0} \rightarrow{ }^{7} \mathrm{~F}_{2}$ transition is very sensitive to the intermediate environments around $\mathrm{Eu}^{3+}$ ions. The synthesized Eu complex exhibited a synergistic effect in which the ligand could absorb UV light and transfer energy to Eu(III), leading to enhancement of intensity.
Variation of emission intensity of complex with $\mathrm{Ph}$ is displayed in Figure 6.

\subsection{Determination of CIE Co-ordinates for Eu(dmh) $)_{3}$ phen}

The CIE chromaticity coordinates of the light emission from these $\mathrm{pH}$ dependent phosphors, excited at 372nm are depicted in Figure 7.

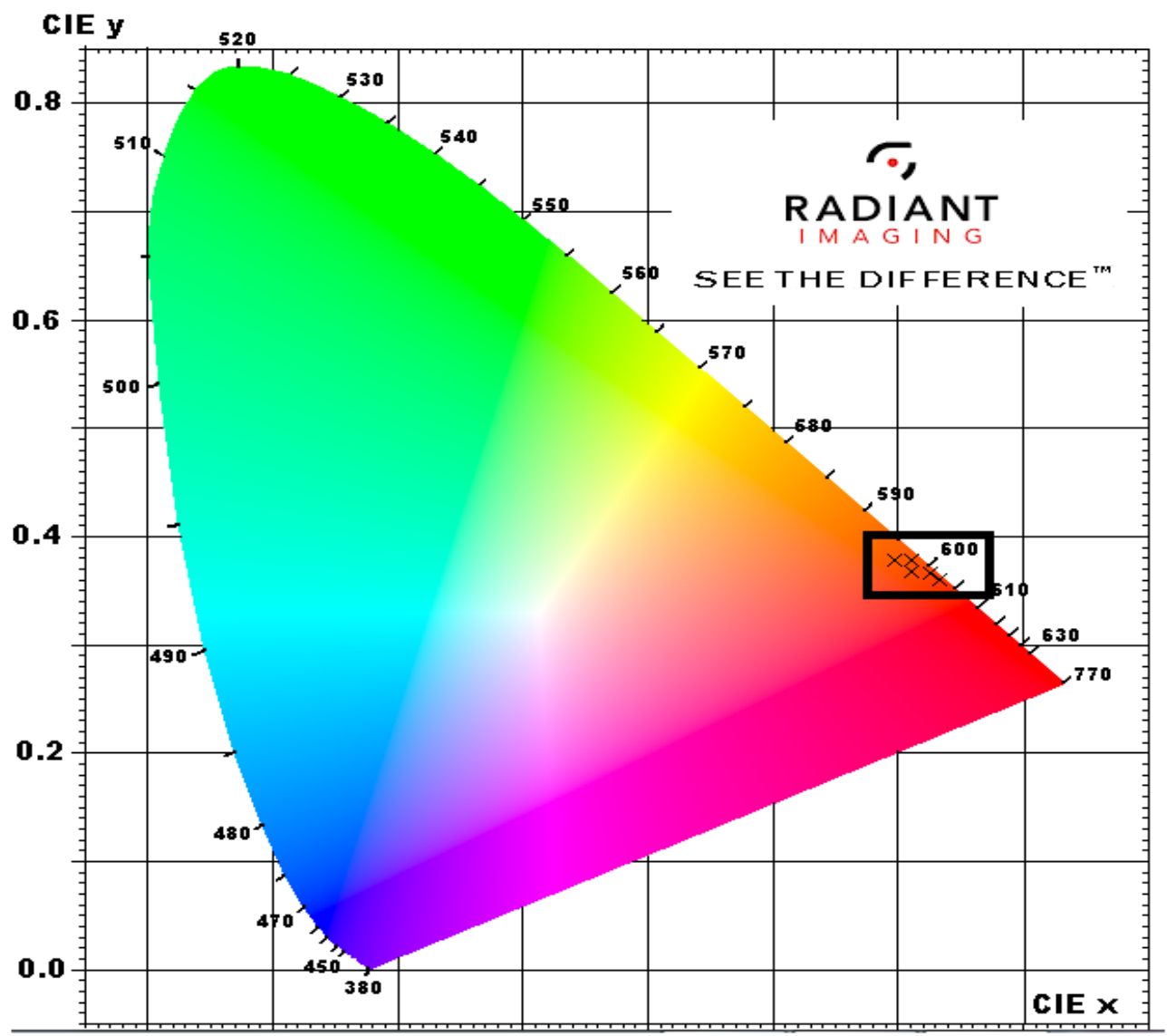

Figure 7. CIE 1931 (x, y) diagram displaying emission color coordinates of $\mathrm{Eu}(\mathrm{dm})_{3}$ phen

Table 1. Yield \% and $\mathrm{X}, \mathrm{Y}$ co-ordinate values in CIE system with varying $\mathrm{pH}$ value of complex $\mathrm{Eu}(\mathrm{dmh})_{3} \mathrm{phen}$

\begin{tabular}{|c|c|c|c|c|}
\hline Complex & pH & Yield \% & CIE co-ordinates $(x, y)$ & Digital photograp \\
\hline \multirow{5}{*}{ 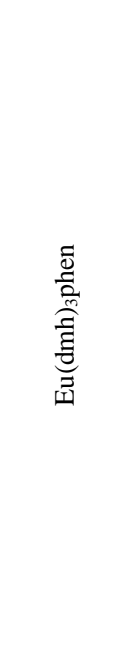 } & 6.0 & $62.8 \%$ & $(0.580,0.326)$ & \\
\hline & 6.5 & $65.0 \%$ & $(0.577,0.325)$ & \\
\hline & 7.0 & $72.0 \%$ & $(0.585,0.327)$ & \\
\hline & 7.5 & $70.4 \%$ & $(0.581,0.325)$ & \\
\hline & 8.0 & $62.1 \%$ & $(0.582,0.324)$ & \\
\hline
\end{tabular}


The emission colour of the synthesized phosphors with different $\mathrm{pH}$ lies in the orange-reddish region with $\mathrm{CIE}$ coordinates varying from $(0.582,0.324)$ to $(0.580,0.326)$. The CIE co-ordinates of $\mathrm{Eu}(\mathrm{dmh})_{3}$ phen at $\mathrm{pH} 6,6.5,7,7.5$ and 8 are found to be $(0.582,0.324),(0.581,0.325)$, (0.585, 0.327), (0.577, 0.325) and (0.580, 0.326), respectively. Starting from the higher $\mathrm{pH}$ value 8.0, it was found that the values of $\mathrm{x}, \mathrm{y}$ chromaticity co-ordinates increased up to $7.0 \mathrm{pH}$, whereas with further decrease in the $\mathrm{pH}, \mathrm{x}$ and $\mathrm{y}$ values dropped down till $6.0 \mathrm{pH}$. However, the largest values of $\mathrm{x}$ and $\mathrm{y}$ co-ordinates are $(0.585,0.327)$ corresponding to $7.0 \mathrm{pH}$. Summary of yield \%, CIE co-ordinates and the digital photographs of raw complexes synthesized at different values of $\mathrm{pH}$ shown in Table 1.

\subsection{Absorption Spectra}

The UV-vis absorption spectra for the synthesized volatile complex Eu(dmh) $)_{3}$ phen was recorded and analyzed with a UV-vis spectrophotometer with PC interface by first calibrating with a cuvet of chloroform/toluene/THF/acetic acid to get a baseline, later, running a cuvet filled with each of the solutions at different molar concentrations. Different solutions of $\mathrm{Eu}(\mathrm{dmh})_{3}$ phen of chloroform, toluene and THF (basic medium) and acetic acid (acidic medium), respectively were prepared with different molar concentrations to study the shift of $\pi \rightarrow \pi^{*}$ and the $\mathrm{n} \rightarrow \pi^{*}$ optical transitions in $\mathrm{Eu}^{3+}$ ion and $\beta$-diketonate moieties. Figure 8 shows the optical absorption spectra of the electro and photo active solvated $\mathrm{Eu}(\mathrm{dmh})_{3}$ phen complex with $\mathrm{pH}=7.0$ for the molar concentration of $10^{-3} \mathrm{~mol} / \mathrm{L}$ in various basic and acidic solvents [10].

The absorption spectra of solvated $\mathrm{Eu}(\mathrm{dmh})_{3}$ phen exhibits two absorption peaks, one at $249 \mathrm{~nm}$ and other in the range of 289-330 nm, which may be attributed to the $\pi \rightarrow \pi^{*}$ and the $\mathrm{n} \rightarrow \pi^{*}$ optical transitions of the $\mathrm{Eu}^{3+}$ ion and $\beta$-diketonate moieties, respectively. Intense absorption peaks are reported for the $10^{-3} \mathrm{M}$ solution. Strong absorption peak at $289 \mathrm{~nm}, 295 \mathrm{~nm}, 307 \mathrm{~nm}$ and 330nm can be observed in UV-vis spectra of $\mathrm{Eu}(\mathrm{dmh})_{3}$ phen in chloroform, THF, toluene and acetic acid, which may be due to $n \rightarrow \pi^{*}$ optical transition. A weak shoulder was accompanied at $249 \mathrm{~nm}$ corresponding to $\mathrm{n} \rightarrow \pi^{*}$ transition for all the organic solvents considered in the present study. The optical densities of the excited molecules in THF and toulene were found to be greater as compared to that of in acetic acid, indicating little decomposition of the ligand in acidic conditions [11]. Hypsochromic shift - a change of spectral band position in the absorption, reflectance, transmittance, or emission spectrum of a molecule to a shorter wavelength (higher frequency) was observed in the optical absorption spectra of $\mathrm{Eu}(\mathrm{dmh})_{3}$ phen, when the solvent is changed from acidic to basic media. This effect is also commonly called a blue shift because the blue colour in the visible spectrum has a shorter wavelength than most other colors. Comparative graph of $\pi-\pi^{*}$ and $n-$ $\pi^{*}$ transitions in absorption spectra of $\mathrm{Eu}(\mathrm{dmh})_{3}$ phen complex in different solvents is shown in Figure 9.

\subsection{X-Ray Diffraction}

Figure 10 shows the diffractogram of $\mathrm{Eu}(\mathrm{dmh})_{3}$ phen and displays many strong, sharp diffraction peaks inferring the crystalline nature of the synthesized complex $100 \%$ relative intensity corresponds to d-value of $9.82 \mathrm{~A}^{0}$ at interplanar distance of $8.99 \mathrm{~A}^{0}$.

\subsection{Scanning Electron Microscopy (SEM)}

The synthesized Eu(TTA) $)_{3}$ bipy complex was analyzed with SEM for topological investigations and the digital images for different resolutions are shown in Figure 11. The obtained micrograph demonstrates the agglomeration of the particles with various grain size. The digital images illustrates that the complex exhibits rod like surface morphology having dimensions in micrometer scale and have crystalline nature as displayed in Figure 11(a, b, c). Thus the data from XRD resembles with the SEM analysis, proving crystallinity of the complex.

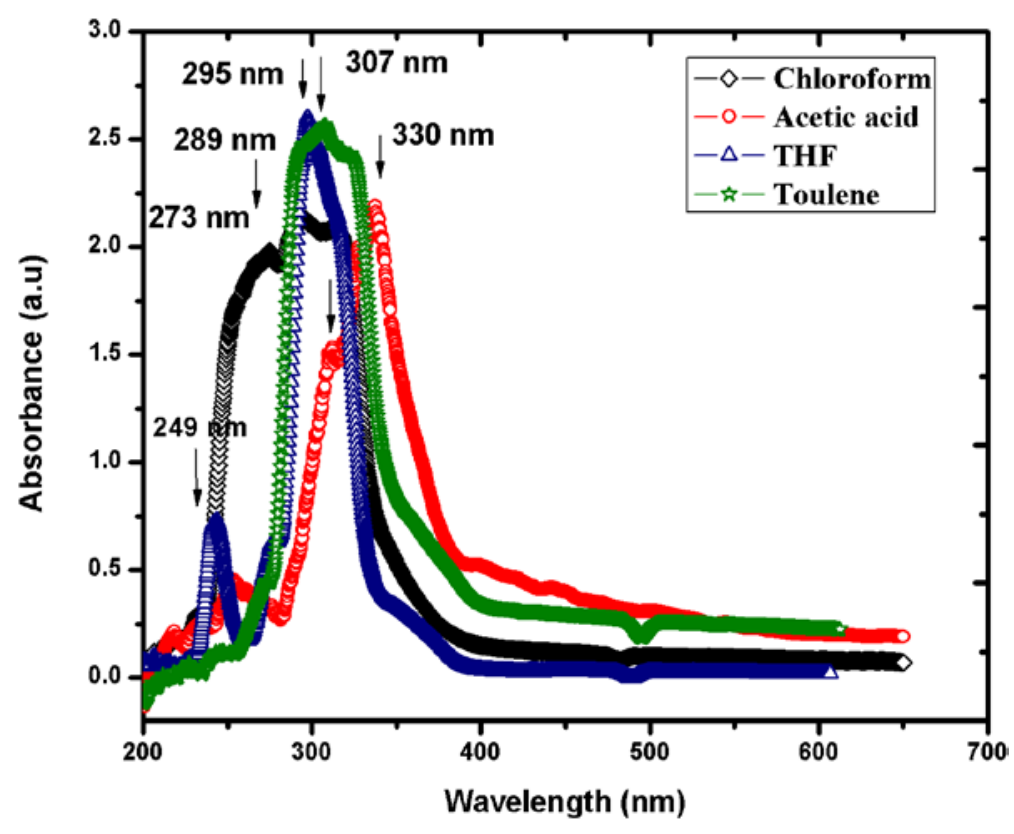

Figure 8. UV-vis spectra of $\mathrm{Eu}(\mathrm{dmh})_{3}$ phen in various solvents 


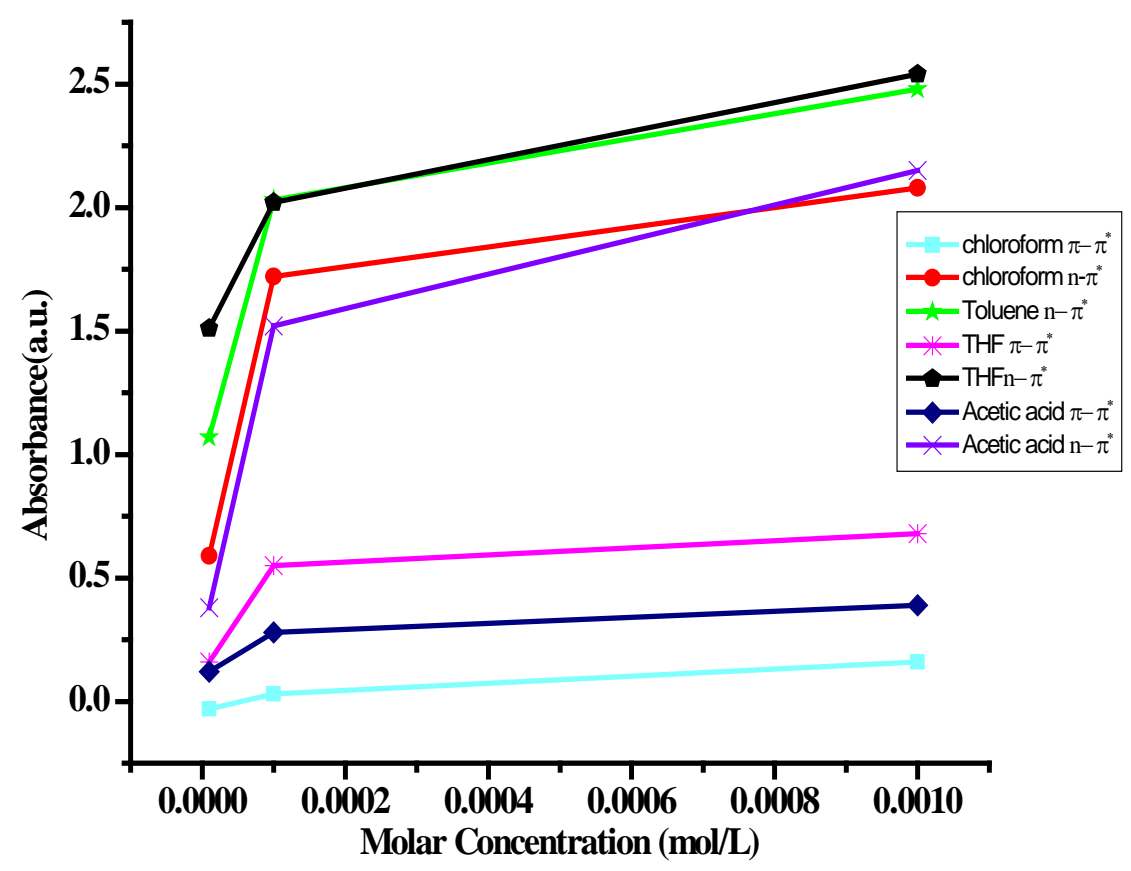

Figure 9. Comparative graph of $\pi$ - $\pi^{*}$ and $n-\pi^{*}$ transitions in absorption spectra of $\mathrm{Eu}(\mathrm{dmh})_{3}$ phen complex in different solvents

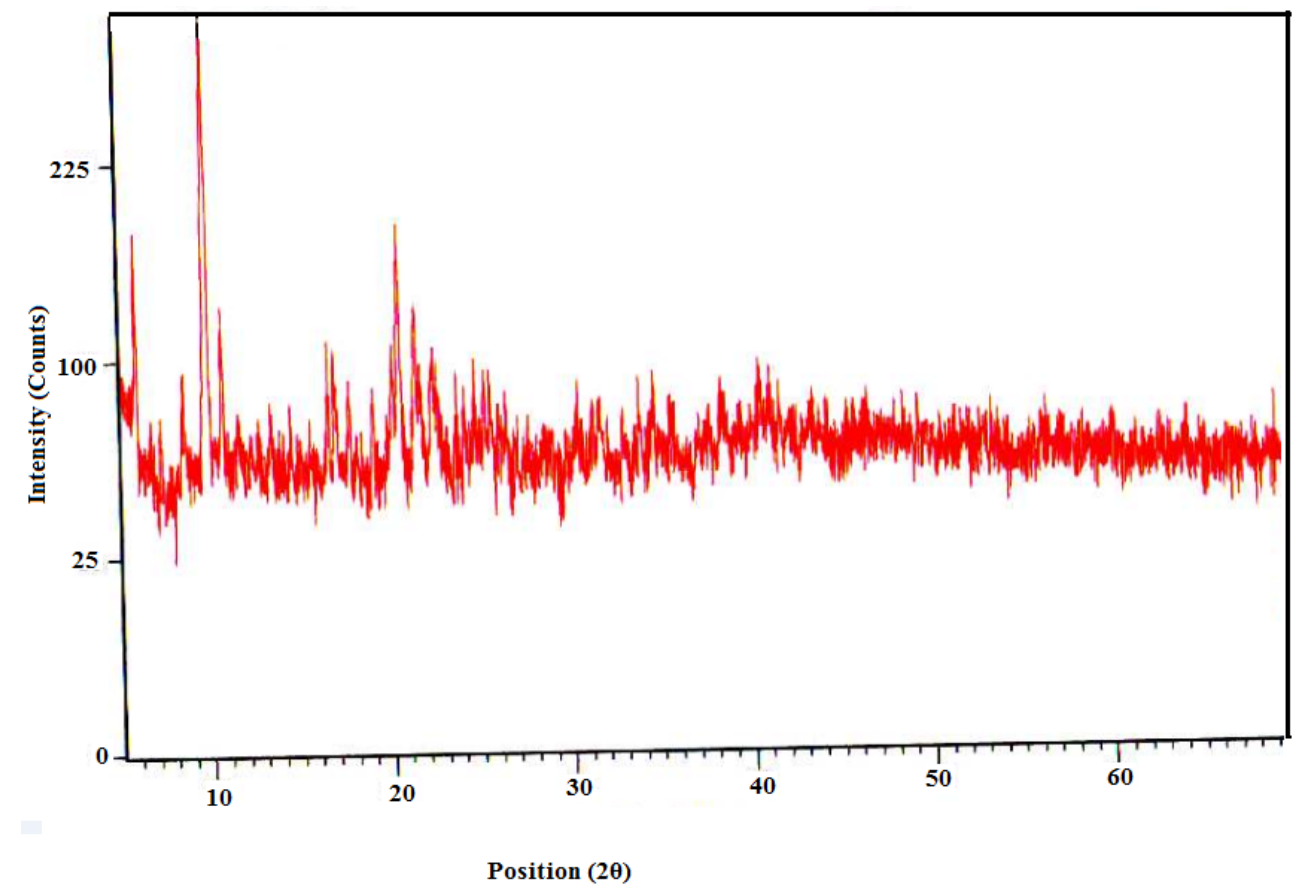

Figure 10. Diffractogram of $\mathrm{Eu}(\mathrm{dmh})_{3} \mathrm{phen}$ at $\mathrm{pH}=7.0$

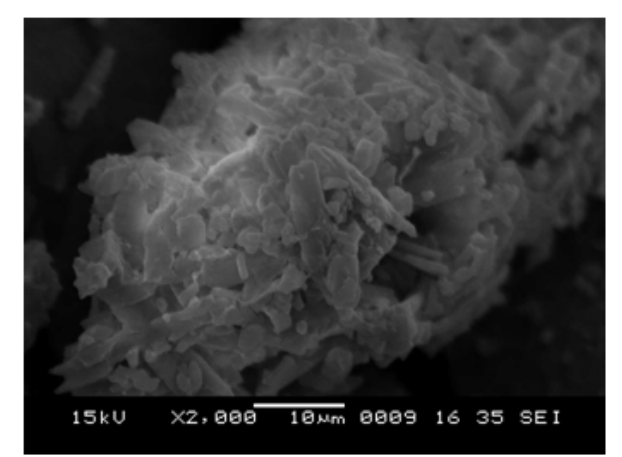

(a)

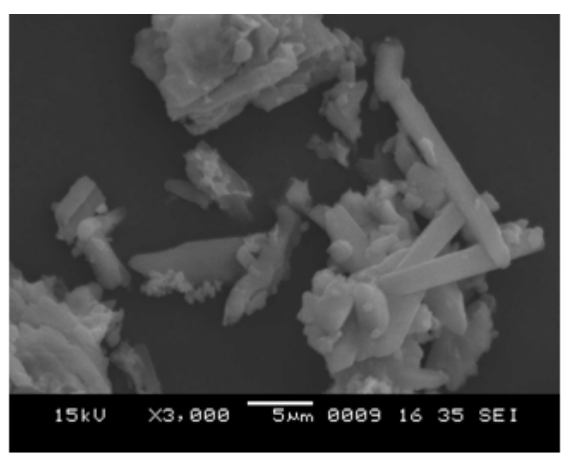

(b)

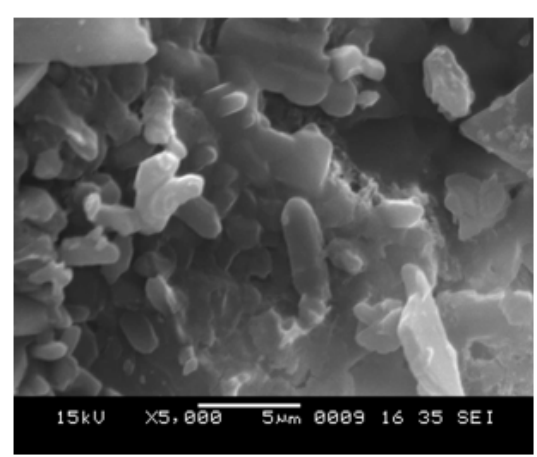

(c)

Figure 11. Digital micrographs of $\mathrm{Eu}(\mathrm{dmh})_{3}$ phen complex at $\mathrm{pH}=7.0$ 


\subsection{Fourier Transform Infrared (FT-IR) Spectra}

The FT-IR spectrum of $\mathrm{Eu}(\mathrm{dmh})_{3}$ phen samples measured on $\mathrm{KBr}$ pellets in a Perkin Elmer-Spectrum Fourier transform infrared (FT-IR) spectrometer with $\mathrm{KBr}$ beam splitters and a resolution of $20 \mathrm{~cm}^{-1}$ is shown in Figure 12. Spectrum shown here is raw data, without any further correction (smoothing, background). In the case of the polycrystalline samples, scattering due to crystallinity is responsible for the broad background with some asymmetric peaks. Broad background with some asymmetric peaks were observed below $450 \mathrm{~cm}^{-1}$, which may be due to scattering of crystalline nature of the phosphor and hence not recorded.

The absorption bands in the finger print region (1600-
$1350 \mathrm{~cm}^{-1}$ ) are generally due to intra molecular phenomena, and are highly specific for each material. They predicts aromatic ring stretching, revealing the presence of $\mathrm{C}=\mathrm{C}$ group. The aromatic $\mathrm{C}-\mathrm{H}$ vibration stretch appears at $2800-3000 \mathrm{~cm}^{-1}$. The strong and considerable peak at $3146 \mathrm{~cm}^{-1}$ predicts the presence of ammonium ion in the complex [12,13]. The aromatic C-C bands emerged about $1500 \mathrm{~cm}^{-1}$. The peak $1965 \mathrm{~cm}^{-1}$ owes to the carbonyl group can be observed in the FTIRspectrum of $\mathrm{Eu}(\mathrm{dmh})_{3}$ phen. Strong peaks between 1600$1400 \mathrm{~cm}^{-1}$ may be due to the presence of $\mathrm{C}=\mathrm{N}$ group. The peaks between $700-1300 \mathrm{~cm}^{-1}$ is indicative of skeletal C$\mathrm{C}$ vibrations. These results confirm the formation of the desired complex and the presence of dmh and phen in the synthesized Eu- $\beta$-diketonate $\mathrm{Eu}(\mathrm{dmh})_{3}$ phen organic hybrid complex.

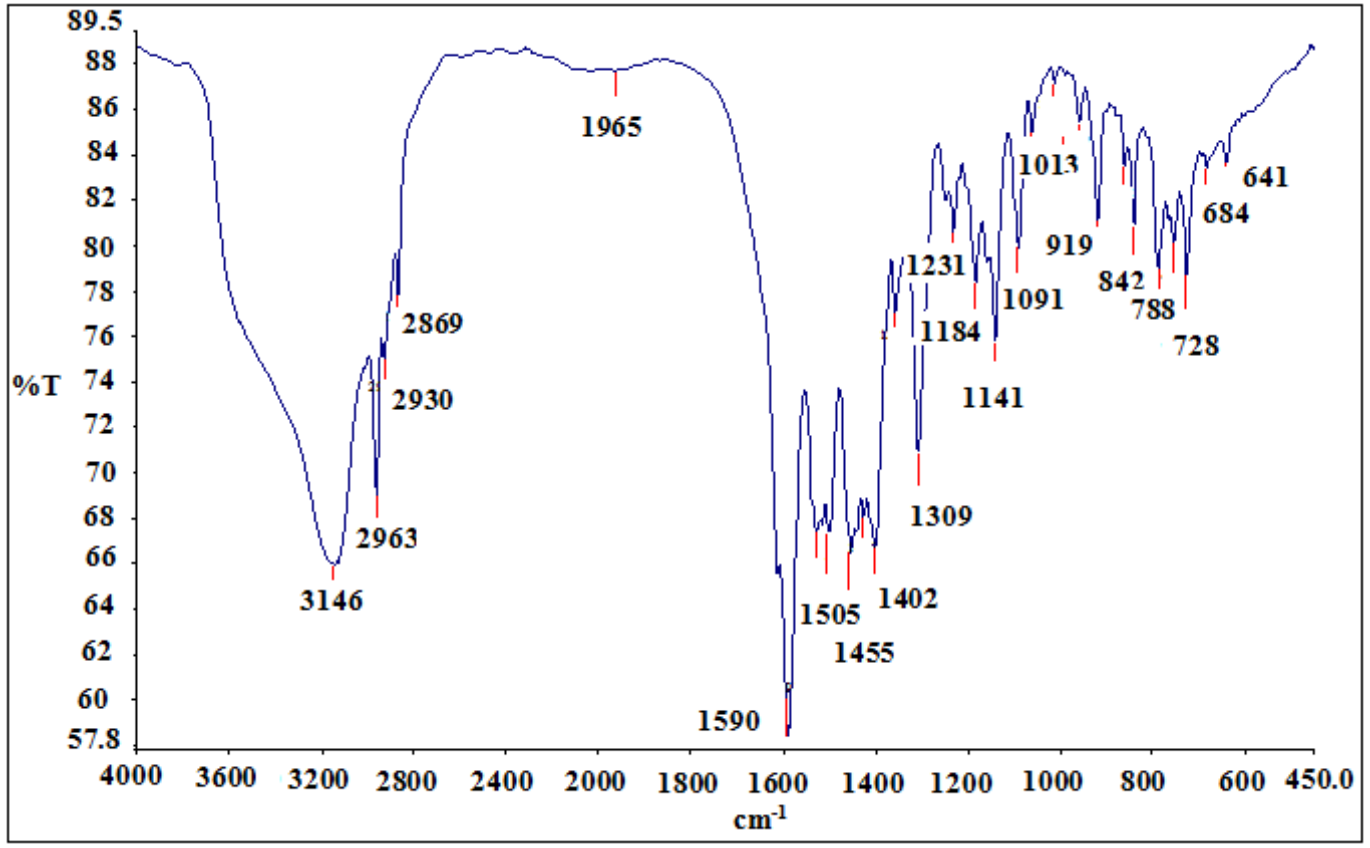

Figure 12. FTIR Spectrum of $\mathrm{Eu}(\mathrm{dmh})_{3}$ phen for $\mathrm{pH}=7.0$

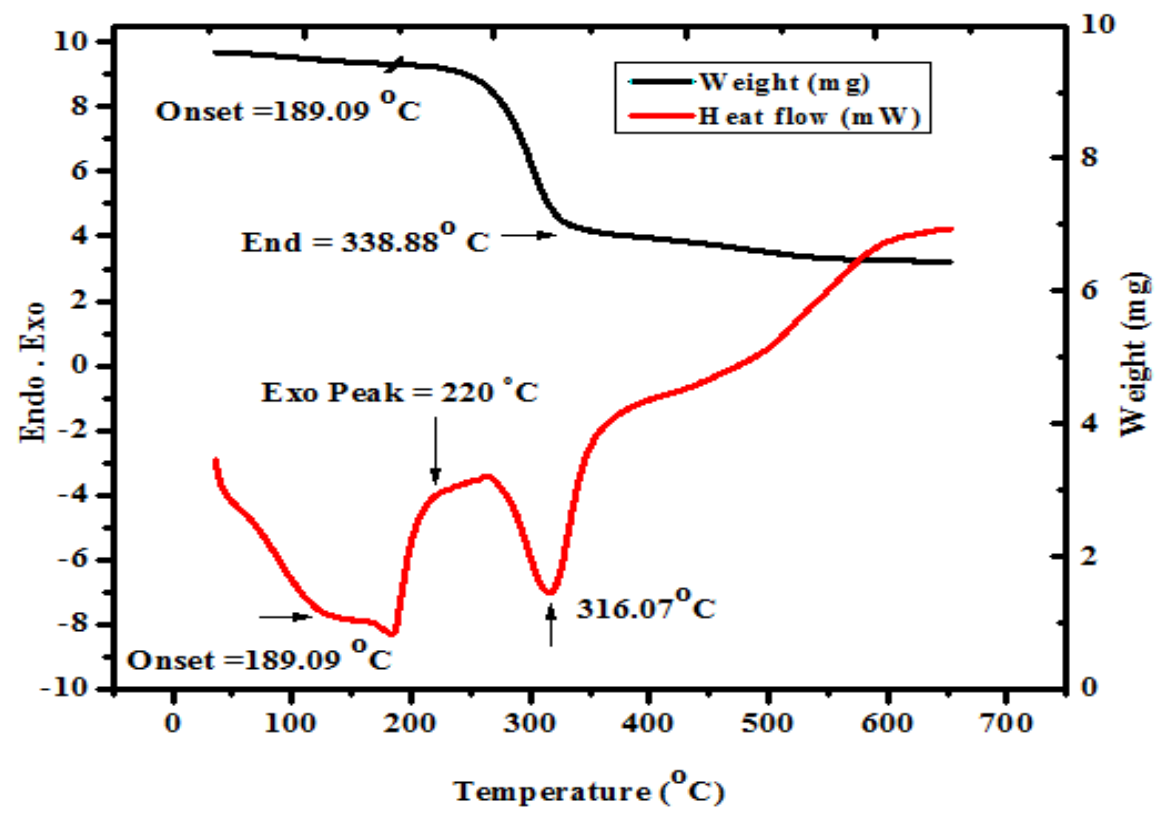

Figure 13. TGA/DTA curve of $\mathrm{Eu}(\mathrm{dmh})_{3}$ phen at $\mathrm{pH}=7.0$ 


\subsection{Thermo Gravimetric and Differential Thermal Analysis (TGA/DTA)}

To elucidate the thermal properties of $\mathrm{Eu}(\mathrm{dmh})_{3}$ phen complex, a TGA/DTA analysis was carried out. The results so obtained are depicted in the thermogram shown in Figure 13. The thermogram revealed no significant loss in the weight of sample until the temperature reached $189.09^{\circ} \mathrm{C}$ which is denoted as the onset point. Beyond the onset temperature a small decrease in the weight of sample started from $220^{\circ} \mathrm{C}$ which can be attributed as the melting point of the complex upto the end point temperature $338.88^{\circ} \mathrm{C}$. No further loss of weight of the synthesized complex is seen from the TGA data. DTA curve of the complex shows two endothermic peaks at $189.09^{\circ} \mathrm{C}$ and $316.07^{\circ} \mathrm{C}$ resulting in the distortion of water molecules and evaporation of residual moisture from the surface of the complex. The exothermic peak at $220^{\circ} \mathrm{C}$ corresponds to the decomposition process of the residual organic materials. No further exothermic peak is found in the DTA results giving strong thermal stability to the complex which is very important for the fabrication of OLED devices, displays and solid state lighting.

\section{Conclusions}

Volatile europium $\beta$-diketonate $\mathrm{Eu}(\mathrm{dmh})_{3}$ phen (Eu: Europium chloride, 2,6-Dimethyl-3,5-heptanedione, 1,10 Phenanthroline)europium(III)complex for different $\mathrm{pH}$ values, ranging between 8.0 and 6.0 were synthesized by precipitation method maintaining stichiometric ratio. The effect of $\mathrm{pH}$ value on the characteristic photoluminescence spectra (PL) was studied for the synthesized complexes. The UV-vis absorption spectra of solvated $\mathrm{Eu}(\mathrm{dmh})_{3}$ phen exhibits two absorption peaks, one at $249 \mathrm{~nm}$ and other in the range of $289-330 \mathrm{~nm}$, which may be attributed to the $\pi \rightarrow \pi^{*}$ and the $\mathrm{n} \rightarrow \pi^{*}$ optical transitions of the $\mathrm{Eu}^{3+}$ ion and $\beta$-diketonate moieties, respectively. Optical densities of the excited molecules in chloroform, THF and toulene were greater as compared to that of in acetic acid, indicating little decomposition of the ligand in acidic conditions. $\mathrm{Eu}(\mathrm{dmh})_{3}$ phen exhibits red intense emission at $613 \mathrm{~nm}$ with a sharp spectral bandwidth of $5 \mathrm{~nm}$ when excited at a wavelength of 372nm with narrow full width at half maxima (FWHM), due to $\mathrm{f}-\mathrm{f}$ transitions of the central $\mathrm{Eu}^{3+}$ ion. Among all synthesized complexes, the complex with $\mathrm{pH} 7.0$ showed maximum peak intensity. However of $\mathrm{pH}$, the position of the emission spectra, corresponding to the ${ }^{5} \mathrm{D}_{0} \rightarrow{ }^{7} \mathrm{~F}_{2}$ electric dipole transition remained at $613 \mathrm{~nm}$ as the $\beta$-diketonate ligand (dmh) used is same in all complexes. Among all the synthesized complexes, the complex with $\mathrm{pH} 7.0$ showed maximum peak intensity, which may be due to superior intermolecular energy transfer between Eu(III) and $\beta$-diketonate ligand. The complex exhibiting maximum intensity was assessed for structural, thermal and luminescence behavior in various solvents. X-ray diffractogram reveals its crystalline nature with $100 \%$ relative intensity corresponding to $\mathrm{d}$-value of $9.82 \mathrm{~A}^{0}$ at interplanar distance of $8.99 \mathrm{~A}^{0}$. The calculated grain size of particle at $100 \%$ relative intensity was found to be $2.76 \mathrm{~nm}$. FTIR spectra confirms the complete formation of the complex with and the presence of dmh and phen structure in the metal complex. TGA/DTA curve of $\mathrm{Eu}(\mathrm{dmh})_{3}$ phen complex reveals that the complex has an ability to maintain its properties unchanged upon heating till $189.09^{\circ} \mathrm{C}$. With further increase in temperature, decomposition of the sample was observed. End peak at $338.88^{\circ} \mathrm{C}$ indicates the final temperature at which the cumulative weight change first reaches its maximum value, corresponding to the complete reaction. Hypsochromic shift was observed in the optical absorption spectra of $\mathrm{Eu}(\mathrm{dmh})_{3}$ phen, when the solvent is changed from basic to acidic media. The effect of change in $\mathrm{pH}$ value of the complex on the characteristic photoluminescence spectra (PL) was studied for the synthesized complexes. The synthesized Eu complex exhibits synergistic effect in which the ligand could absorb UV light and transfer energy from Eu(III), leading to enhancement of intensity, suggesting a potential application of the complexes as red emitting materials in OLEDs, display devices and solid state lighting.

\section{Acknowledgements}

One of the authors (NTK) wishes to thank Rashtrasanth Tukadoji Maharaj Nagpur University for providing financial support to carry out the synthesis and characterization of the synthesized Eu(TTA) 3 bipy complex under University Research Project Scheme (Sanction letter No. Dev. 2140 dated $16^{\text {th }}$ Nov. 2015).

\section{References}

[1] N. Thejo Kalyani, S.J. Dhoble, "Importance of Eco-friendly OLED Lighting," Defect and Diffusion Forum, Trans Tech Publications, Switzerland 357, (2014), 1-27.

[2] N A.H. Male, Oleg V.Salata, Victor Christou, "Enhanced electroluminescent efficiency from spin-coated europium(III) organic light-emitting device”, Synth metals 12, (2002) 7-10.

[3] H Liang, Fang Xie, "Photoluminescence study of a europium (III) complex containing 1, 5-styrylacetylacetone ligands”, Spectrhema Acta Part A, 77, (2010) 348-350.

[4] L Zhang, Bin Li, "A series of Eu(III) emitters with a novel triphenylamine-derived beta-diketone ligand,” J Lumin 129(11), (2009) 1304-1308.

[5] H Liang, Fang Xie, Xiaojun Ren, Yifa Chen, Biao Chen, Fuquan Guo. "Temperature dependent luminescence of a europium complex incorporated in poly(methyl methacrylate", Spectrochem Acta Part A: Mol Bimol Spectro; 116, (2013) 317-320.

[6] Melby, L. R. Rose, N. J. Abramson, E. Caris, J. C. "Synthesis and fluorescence of some trivalent lanthanide complexes," J. Am. Chem. Soc., 86, 5117, (1964).

[7] S Linfang, Li Yanwei, Cao Huaru, Guan Ying, Ma Yongchun, "Synthesis of novel europium complexes and their photoluminescence properties”, J. Rare Earths, 30(1), (2012) 17-20.

[8] M. Fernandes, V. de Zea Bermudez, R. A. Sa' Ferreira, L. D. Carlos, A. Charas, J. Morgado M. M. Silva,M. J. Smith,” Highly Photostable Luminescent Poly( $\varepsilon$-caprolactone)siloxane Biohybrids Doped with Europium Complexes,” Chem. Mater., 19(16), (2007). 3892-3901.

[9] N. ThejoKalyani, S.J.Dhoble, "Novel materials for fabrication and encapsulation of OLEDs” Ren.Sus.Ene. Rev. 44, (2015) 319-347.

[10] N.Thejo Kalyani, S.J. Dhoble and R.B. Pode. "Optical Properties of $\mathrm{Eu}_{\mathrm{x}} \operatorname{Re}_{(1-\mathrm{x})}(\mathrm{TTA})_{3}$ Phen Organic Complexes in Different Solvents" J. Kor Phys Soc. 57( 4), (2010) 746-751.

[11] K. E, Fukuda .T, Kato .S, Honda .Z, Kamata. N. "pH and concentration dependence of luminescent characteristics in glass- 
encapsulated Eu-complex”, J Sol-Gel Sci Technol, 50(3), (2009) 409-414.

[12] J.P.Coates, Á Review of sampling Methods For Infrared spectroscopy'in Applied Spectroscopy: a compact Reference for practitioners, eds. J.Workman A.W.Sprinsteen, Academic press New York, (1998) 49-91.

[13] J.P.Coates, 'Ínto the unknown: the first in the series', Spectroscopy, 11(5), (1996) 32-39. 